\title{
New Low Voltage Scanning Transmission Electron Microscope Detector for Fastest Image Acquisition in BF, DF and HAADF
}

\author{
R. Salzer, J. Ackermann, R. Arnold, S. Meyer, C. Kübler \\ Carl Zeiss Microscopy, Carl-Zeiss-Straße 22, Oberkochen, 73447, Germany
}

Scanning transmission electron microscopy (STEM) is widely used and common method in the scanning electron microscope world to investigate thin lamellas. Due to the reduced interaction volume of the primary electron beam with the sample it is possible to investigate structures with the highest resolution achievable with the SEM. To save expensive TEM time STEM investigations in a FE-SEM or CrossBeam ${ }^{\circledR}$ are a cost and time efficient alternative when the high resolution imaging capabilities of a TEM are not necessary. The structural investigation of cells and cell structures is good example were STEM imaging in a SEM is an appropriate substitute for a TEM. STEM investigations deliver sufficient resolution and similar contrasts as known from TEM images, whereas the used the tool (i.e. a FIB/SEM) is much more flexible than an expensive TEM. A CrossBeam ${ }^{\circledR}$ system is the perfect tool for such an investigation because it enables you to reliably prepare an ultra-thin TEM lamella that has a certain thickness and highest stability using the $\mathrm{X}^{2}$ method [1] and SmartEPD [2]. And it gives you the ability to do a target preparation whether the region of interest is on the surface or is located several $100 \mu \mathrm{m}$ inside your sample [3].

In cooperation with Merli and Morandi et al. [4,5] a new STEM detector has been developed. This semiconductor based detector consist of multiple segments: a bright field area in the middle, four dark field (DF) diodes, an additional annular dark field (ADF) ring and a dedicated high angle annular dark field (HAADF) outer ring (Figure 1A). The total detector area is $200 \mathrm{~mm}^{2}$ which corresponds to an acceptance angle of up to one rad at minimal detector sample distance (Figure 1B). However, Morandi et al [5] showed that not only a well-known detection setup but also sample properties like the massthickness distribution which can be determined by SmartEPD [2] are fundamental information for a correct interpretation of STEM results.

A tailored fabrication process was used to achieve a higher low voltage responsitivity of the diode (Figure 1C). STEM investigations are no longer limited to energies above $10 \mathrm{kV}$ but may be performed with landing energy lower than $1 \mathrm{keV}$. Whereas it is known that working in this low landing energy range requires excellent preparation of ultra-thin TEM lamella [1,2].

In order to reduce beam driven damage, the acquisition time for an image with an acceptable signal to noise ratio at low beam currents is the major value for a STEM detector. Especially for biological samples - where STEM investigations in a SEM become more and more important - it is necessary to achieve at small probe currents fast acquisition speeds to avoid bleaching of the region of interest. The same principles that enables the achieved sensitivity at low energies enhances the overall efficiency of the detector. This and the diode segment design increases the possible recording speed by a factor of up to eight (compared to established commercial solutions).

This contribution will introduce the new STEM detector. Applications from life (i.e. Figure 2) and material science will emphasize the low voltage performance of the detector, its fast imaging capabilities. An outlook of further developments will be given, i.e. the parallel acquisition of four different STEM settings to decrease the recording time.

References:

[1] L. Lechner et al, Microsc. Microanal. 18, 379-384, 2012 
[2] R. Salzer et al, Microsc. Microanal. 18 (Suppl. 2), 654 (2012)

[3] R. Salzer et al, Microsc. Microanal. 18 (Suppl. 2), 636 (2012)

[4] P.G. Merli and V. Morandi, Mic. Mic. 11 (2005), 97

[5] V. Morandi and P.G. Merli, J. App. Phys. 101(2007), 114917

A)

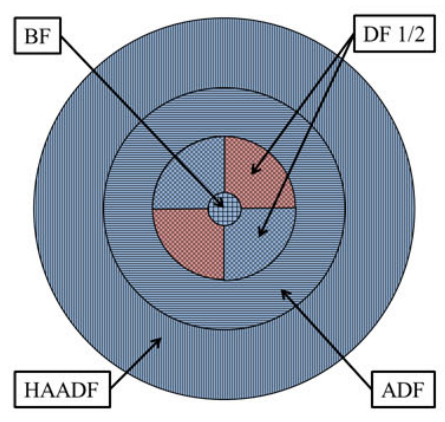

B)

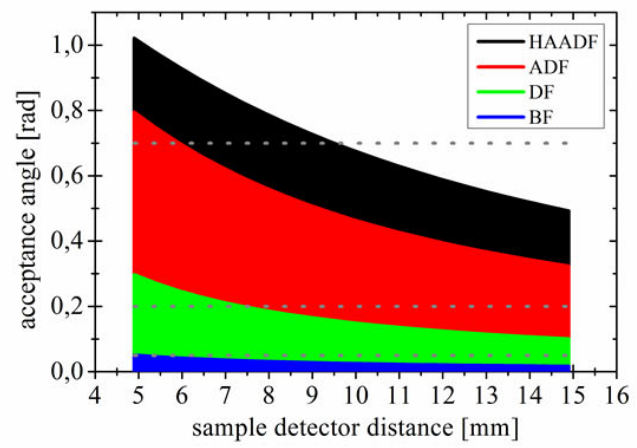

C)

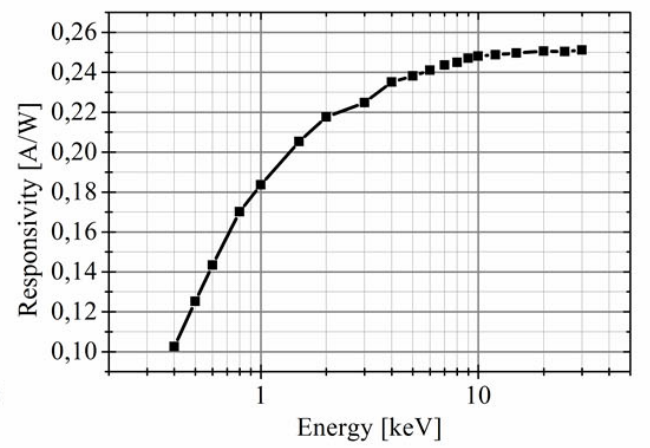

Figure 1. A) Scheme of the different detector segments that can be selected for imaging. Each two of the four dark field segments are connected to one DF detector segment. B) Possible acceptance angles in dependency of the sample detector distance shown for the different segments. Dotted lines marking the typical values for bright, dark and high angle annular dark field. C) Responsivity curve of the diode in dependence on the electron landing energy.

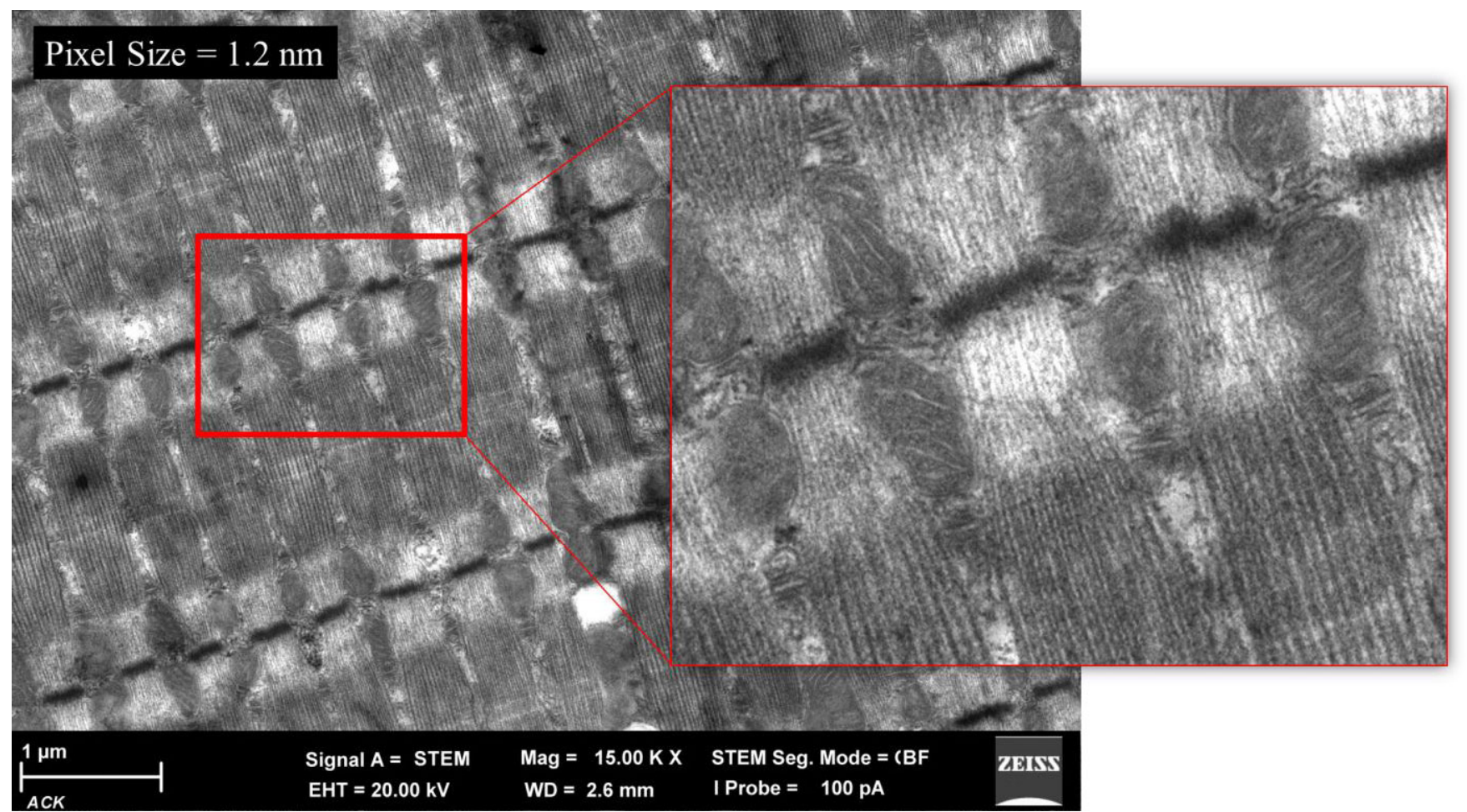

Figure 2. Bright field image of a heart muscle cell (courtesy from Prof. Dr. Kirfel, University of Bonn) of a mice taken at $20 \mathrm{keV}$ primary electron energy and with a resolution of 6144 x 4608 pixel whereas the pixel size is $1.2 \mathrm{~nm}$ taken in $15.3 \mathrm{~min}$. 